\title{
The impact of monomer sequence and stereochemistry on the swelling and erosion of biodegradable poly(lactic-co-glycolic acid)matrices
}

Michael A. Washington ${ }^{\mathrm{a}}$, Devin J. Swiner ${ }^{\mathrm{a}}$, Kerri R. Bell ${ }^{\mathrm{a}}$, Morgan V. Fedorchak ${ }^{\mathrm{b}, \mathrm{g}, \mathrm{g}, \mathrm{h}}$, Steven R. Little $^{\mathrm{b}, \mathrm{c}, \mathrm{d}, \mathrm{e}, \mathrm{f}, \mathrm{g}}$, Tara Y. Meyer ${ }^{\mathrm{a}, \mathrm{g}, *}$

${ }^{\mathrm{a}}$ Department of Chemistry, ${ }^{\mathrm{b}}$ Chemical and Petroleum Engineering, ${ }^{\mathrm{c}}$ Ophthalmology, ${ }^{\mathrm{d}}$ Bioengineering, ${ }^{\mathrm{e}}$ Immunology, ${ }^{\mathrm{f}}$ Pharmaceutical Sciences, ${ }^{\mathrm{g}} \mathrm{McG}$ cwan Institute for Regenerative Medicine, ${ }^{\mathrm{h}}$ Fox Center for Vision Restoration, University of Pittsburgh, Pittsburgh, PA 15260 USA

*Corresponding author. Current address: Department of Chemistry, University of Pittsburgh, 219 Parkman Avenue, Pittsburgh, PA 15260, USA. Tel.: 412-624-8635; fax: 412-624-8611.e-mail address: tmeyer@pitt.edu (Tara Y. Meyer)

\begin{abstract}
.
Monomer sequence is demonstrated to be a primary factor in determiningthe hydrolytic degradation profile of poly(lactic-Co-glycolic acid)s (PLGAs). Although many approaches have been used to tune the degradation of PLGAs, little effort has been expended in exploring the sequence-control strategy exploited by nature in biopolymers. Cylindrical matrices and films prepared from a series of sequenced and random PLGAs were subjected to hydrolysis in a $\mathrm{pH}$ 7.4 buffer at $37^{\circ} \mathrm{C}$. Swelling ranged from $107 \%$ for the random racemic PLGA with a 50:50 ratio of lactic (L) to glycolic $(\mathrm{G})$ units to $6 \%$ for the sequenced alternating copolymer poly LG. Erosion followed an inverse trend with the random 50:50 PLGA showing an erosion half-life of 3-4 weeks while poly LG required ca. >10 weeks. Stereosequence wasfound to play a large role in determining swelling and erosion; stereopure analogs swelled less and were slower to lose mass. Molecular weight loss followed similar trends and increases in dispersity correlated with the onset of significant swelling.The relative proportion of rapidly cleavable G-G linkages relative to G-L/L-G (moderate) and L-L (slow) correlates strongly with the degree of swelling observed and the rate of erosion. The dramatic sequence-dependent variation in swelling, in the absence of a parallel hydrophilicity trend, suggest that osmotic pressure, driven bythe differential accumulation of degradation products, plays an important role.
\end{abstract}

Keywords.PLGA; biodegradable polymers; polymer swelling;polymer erosion; sequenced copolymers; polymer degradation 


\section{Introduction}

We have discovered that sequence can be used to control the degradation behavior of poly(lactic-co-glycolic acid)s (PLGAs). Random PLGAs have been utilized extensively in controlled drug delivery systems and clinical applications dueto their biocompatibility, biodegradability, and regulatory acceptance[1].Target applications includecontrolled release devicesfor anticancer agents[2-4], surgical sutures and screws [5, 6], and porous scaffolds for tissue regeneration [7].Despite the number of literature reports on the utility of PLGAs for bioengineering, however, there are currently only 15 FDA-approved PLA/PLGA-based drug products on the US market [8]. We hypothesize that one contributing factor to the poor translation into application is the relatively narrow range of performance of the random copolymer PLGAs during degradation, a deficiency that has been addressed by others with a variety of strategies including adjusting molecular weight, controlling ratio of lactic (L) and glycolic $(\mathrm{G})$ units; tuning average $\mathrm{L}$ and $\mathrm{G}$ block lengths, adding comonomers and chemical additives, and through the design and configuration of devices[9, 10].

Our approach to expanding the accessible range of degradation profiles is to move beyond random copolymers through the control the sequence of the monomers, an approach which in biological polymers has been shown to provide diverse structure and function from a limited monomer set. The potential for impact of this approach is further supported by the recent promising reports of sequence control in other synthetic copolymers [11-19] and by intriguing studies by Sarasuaet al., that link properties with statistical variations in chain microstructure for selected biopolyesters [20-25].

To probe the relationship between monomer order and properties, we have developed a synthetic route that yields PLGAswith repeating sequences[26]. For example, we have preparedpolyLG, which consists of an exact repeat of the LG dimer for the length of the polymer, i.e., $(\mathrm{LG})_{\mathrm{n}}$. Similarly, we have been able to prepare a variety of polymers bearing periodic repeats of varying lengths, e.g., (LLG) $)_{n},(G L L G)_{n}$, etc. Using NMR spectroscopy, which is uniquely powerful for the characterization of this particular class of polymer due to solution phase conformational preferences, theperiodic structure of these polymers has been confirmed. Moreover, sequence errors are easily detected and can be quantified [26].The ability to both synthesize exact sequences and verify them enables, for the first time, a thorough exploration of structure and function in PLGAs.

In initial properties studies of this new class ofperiodic PLGAs [27, 28] we have found a surprisingly strong correlation between sequence and properties. In microparticlesprepared from these copolymers, we observed a correlation of sequence with molecular weight loss, lactic acid release, and thermal properties. In general, the sequenced PLGAs exhibited a slower and more gradual loss of molecular weight and a longer preservation of morphology, including $T_{g}$, than the random analogs. Differences were also observed between sequences, both structural- and stereoisomers. The release of an encapsulated guest molecule, rhodamine B, was also studied and found to depend directly on monomer order; the simple alternating copolymerpoly LGreleased the guest more slowly and gradually than did the random PLGA control. 
Based on these results, we hypothesize that sequenced PLGAs may have positive implications for bioengineering applications where prolonged delivery timesand/or structural integrity are particularly important. For example, a longer release time would be beneficial for long-lasting intraocular implants. These implants, which are designed to replace eye drop regimens, which have low patient compliance, allow for continuous drug release [29]. We also expect that the slower degradation of the sequenced PLGAs will minimize the accumulation of $\mathrm{pH}$-lowering acidic by-products compared to random PLGAs. This behavior could provide a protective effect for tissues such the retina which is known to beparticularly sensitive to nonphysiological $\mathrm{pH}$ [30]. The improved maintenance of morphology for the sequenced PLGAs could also offer advantages for certain applications, e.g., the repair of craniofacial bony defects, which require longer term mechanical strength during the delivery of critical osteogenic growth factors [31].

Swelling and erosion are deeply relevant to these application goals and their study should also lend insight into any sequence-based differences in the underlying mechanism of erosion. It has been previously reported and substantiated by numerous studies that matrices of random PLGAs below certain dimensions degrade by bulk erosion [32, 33]. Observable behaviors associated with this mechanism include, in most cases, significant swelling and a rapid loss of mass after an initial latent period. At the other end of the continuum lie materials that decompose by surface erosion. These materials, e.g., polyanhydrides,degrade without significant water uptake and mass loss is gradual as the inner layers only begin to degrade as the outer layers are sequentially hydrolyzed [34-38]. This distinction is particularly important for the use of PLGA in drug delivery, as it is expected and has been observed that the release profile of encapsulated drugs depends on swelling and erosion [39].Herein, we examine the swelling and erosion of sequenced PLGAs to determine, in part,whether their degradation behavior aligns more closely with a bulk or surface mechanism.

\section{Materials and methods}

\subsection{Materials}

Periodic PLGA copolymers were prepared as previously described[26, 40]. Poly(D,L-lactideco-glycolide) with a 50:50 ratio of lactic to glycolic acid-derived units and carboxylate end groups(PDLGA-50) and poly(D,L-lactide-Co-glycolide) with a 65:35 ratio of lactic to glycolic acid-derived units and carboxylate end groups(PDLGA-65) were obtained from Durect Corporation (Birmingham, AL) as a pelletized solid. Prior to use, the polymers were dissolved in methylene chloride $\left(\mathrm{CH}_{2} \mathrm{Cl}_{2}\right)$ and precipitated in methanol to yield offwhite amorphous solids. Poly(L-lactide-co-glycolide) with a 50:50 ratio of lactic to glycolic acid-derived units and carboxylate end groups (PLLGA-50), was obtained from Changchun SinoBiomaterials Co. Ltd. (Changchun, China) as a fibrous white solid and was used as provided. Phosphate buffered saline (PBS, $\mathrm{pH}=7.4,10 \mathrm{mM}$ ) was purchased from Life Technologies (Carlsbad, CA). 


\subsection{Characterization}

Molecular weights and dispersities were acquired on a Waters GPC system (THF, 0.5 $\mathrm{mL} / \mathrm{min}$ ) with Jordi $500 \AA, 1000 \AA$, and $10000 \AA$ divinylbenzene (DVB) columns and refractive index detector (Waters) which was calibrated relative to polystyrene standards. Thermal properties of all polymers were obtained using TA Instruments Q200 DSC. Standard data were collected with a heating and cooling rate of $10^{\circ} \mathrm{C} / \mathrm{min}$. Melting transitions $\left(\mathrm{T}_{\mathrm{m}}\right)$ were collected from the first heating cycle and glass transition temperatures $\left(\mathrm{T}_{\mathrm{g}}\right)$ were collected during the second heating cycle. Inflection points of glass transition temperatures are reported. All samples were prepared by drop-casting $\left(\mathrm{CH}_{2} \mathrm{Cl}_{2}\right)$ into DSC pans followed by vacuum drying for $24 \mathrm{~h}$ and annealing at $85{ }^{\circ} \mathrm{C}$ for $3 \mathrm{~h}$. The ${ }^{1} \mathrm{H}$ and ${ }^{13} \mathrm{C}$ NMR spectra were obtained in $\mathrm{CDCl}_{3}$ using a 500 $\mathrm{MHz}$ Bruker spectrometer at $293 \mathrm{~K}$ and calibrated to the residual solvent peak at $\delta 7.26 \mathrm{ppm}\left({ }^{1} \mathrm{H}\right)$ and $\delta 77.00 \mathrm{ppm}\left({ }^{13} \mathrm{C}\right)$.Matrix assisted laser desorption/ionization time-of-flight (MALDI-ToFMS) spectra were obtained on a Voyager-DE PRO instrument with a $337 \mathrm{~nm} \mathrm{~N}$ laser and $25 \mathrm{kV}$ accelerating voltage. The mass spectra of the polymers were obtained in reflector mode. The matrix consisted of trans-2-[3-(4-tert-butylphenyl)-2-methyl-2-propenylidene]malonitrile (DCTB) and sodium trifluoroacetate as the cationization agent.

\subsection{Preparation of cylindrical-shaped pellets}

Cylindrical-shaped pellets of all PLGAs were prepared by heated compression molding in a custom press. The colorless polymer (20-30 mg) was loaded into the press which was warmed to 85-95 ${ }^{\circ} \mathrm{C}$ and compressed with a 1,200-1,400 lb load for $10 \mathrm{~min}$ using a Carver press (Hydraulic unit model \#3912; Wabash, IN). The press and sample were then re-heated for $10 \mathrm{~min}$ in an oven at $85^{\circ} \mathrm{C}$, and compressed again for 5 min under the same temperature and load. The resulting pellets were opaque or translucent depending on the polymer used and had dimensions of 3 x $3 \mathrm{~mm}$ (swelling) or 3 x $1.5 \mathrm{~mm}$ (erosion), corresponding to a weight of $\sim 26 \mathrm{mg}$ and $\sim 15$ mg, respectively.

\subsubsection{In vitro swelling of sequenced and random PLGAs}

Twosamples of each polymer (26 mg each) were placed in separate Eppendorf tubes containing $2 \mathrm{~mL}$ of PBS. All tubes were incubated at $37^{\circ} \mathrm{C}$ on a rotating mixer (8 rpm). Samples were removed every $2 \mathrm{~d}$ for the first $10 \mathrm{~d}$, then weekly depending on the degree of swelling. Both PDLGA-50 and PLLGA-50 were removed every 2d until sample degradation progressed to the point that the remaining material could not be handled or weighed.Each sample was blotted dry for $30 \mathrm{~s}$ and weighed on an analytical balance $\left( \pm 1.0 \times 10^{-4} \mathrm{~g}\right)$ until a stable reading was displayed for $15 \mathrm{~s}$. The phosphate buffer was replaced each week for the entirety of the study.

\subsubsection{In vitro erosion of sequenced and random PLGAs}

Ten samples of each polymer (15 mg each) were placed in separate Eppendorf tubes containing $2 \mathrm{~mL}$ of PBS. Samples of each polymer were then divided into 5 groups of 2 samples, each to determine the mass loss at 5 different time intervals. All tubes were incubated at $37^{\circ} \mathrm{C}$ on a rotating mixer $(8 \mathrm{rpm})$. The tubes were refilled with fresh PBS $(2 \mathrm{~mL})$ every week. At each 
time point, duplicate samples of each polymer were collected, blotted dry for $15 \mathrm{~s}$, flash-frozen in liquid $\mathrm{N}_{2}$,then lyophilized for $2 \mathrm{~d}$. After freeze-drying, the pellets were weighed on an analytical balance $\left( \pm 1.0 \times 10^{-4} \mathrm{~g}\right)$. Masses were recorded once a stable reading was displayed for $15 \mathrm{~s}$.

\subsection{Water contact angle experiments}

Water contact angle measurementswere recordedusing a VCA optima XE video contact angle system at $24{ }^{\circ} \mathrm{C}$ and $42-48 \%$ relative humidity. A droplet with a volume of $1 \mu \mathrm{L}$ was formed at the end of the needle and then lowered carefully until contact was made with the sample. The needle was withdrawn immediately so that the droplet was left on the sample surface. An image of the droplet was acquired with a charge-coupled device (CCD) camera $5 \mathrm{~s}$ after contact with the surface of the film. The static contact angle was calculated automatically by the VCA software. Approximately $45 \mathrm{~s}$ was required to complete the whole measurement process. Each measurement was repeated $5 x$ per sample at different locations.

Films of all PLGAs were prepared using a drawdown coating method. Each of the copolymer samples was dissolved in dichloromethane at a concentration of $250 \mathrm{mg} / \mathrm{mL}$. A 20-30 $\mu \mathrm{m}$ film was deposited on a glass microscope slide using a 180-200 $\mu \mathrm{L}$ aliquot of the polymer solution. The resulting film was dried in an oven at $70{ }^{\circ} \mathrm{C}$ for $1 \mathrm{~h}$ and stored in a vacuum desiccator until used. Samples were exposed to PBS at $23^{\circ} \mathrm{C}$ and the hydrated contact angle was monitored over an $8 \mathrm{~d}$ time period. After the hydrated water contact angle was recorded, the films were flashfrozen in liquid $\mathrm{N}_{2}$, lyophilized for $3 \mathrm{~d}$, and the lyophilized film contact angle was determined using the same method.

\section{Results}

\subsection{Naming conventionsand characterization of PLGA copolymers.}

The L-lactic unit, racemic lactic unit, and glycolic unit are abbreviated as $\mathbf{L}, \mathbf{L}_{\text {rac }}$, and $\mathbf{G}$, respectively. Theperiodic copolymers utilized in this study were prepared using segmer assembly polymerization(SAP). In this method, we prepare well-defined oligomers and polymerize them using condensation conditions that have been optimized to preclude sequence scrambling by transesterification(Scheme S1). The resulting copolymers are termed periodic copolymers because they consist of a nearly perfect repetition of the input segmer.As such, a segmer consisting of a lactic and glycolic acid-derived unit, would be termed LG and the polymer would be named poly $\mathbf{L G}$ (Scheme 1). The SAP method was also used to prepare a random copolymer, R-SAP, by condensation of a 1:1:1:1 ratio of the segmers $\mathbf{L G , ~} \mathbf{G L}, \mathbf{L L}$ and GG. The random copolymers, PLLGA-50, PDLGA-50 and PDLGA-65, prepared by ringopening polymerization (ROP) of lactide and glycolide, were purchased. In this case, the PLLGA-50 and PDLGA-50 are the stereopure and racemic versions of the copolymers with a 50:50 L:G-ratio and PDLGA-65 is the racemic derivative of a copolymer with a 65:35 L:G-ratio. Sequenced copolymers prepared by SAP range in molecular weight from 18 to $30 \mathrm{kDa}$ and are comparable to purchased random PLGA controls (Table 1, Figure S4). All polymers exhibited $\mathrm{T}_{\mathrm{g}} \mathrm{in}$ the range of 44 to $49{ }^{\circ} \mathrm{C}$ (Table 1 , Figure S5). 
Synthesis of Sequenced PLGAs

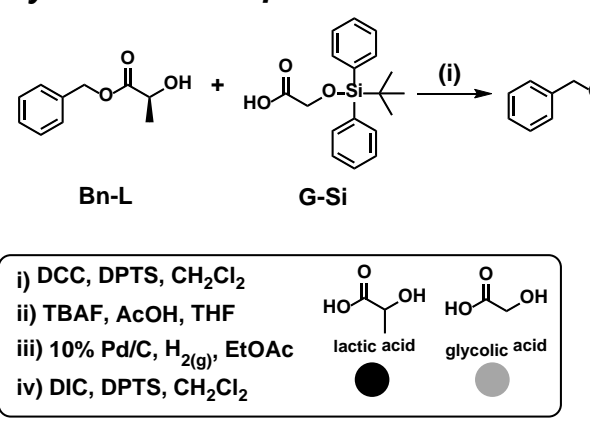

Synthesis of Random PLGAs

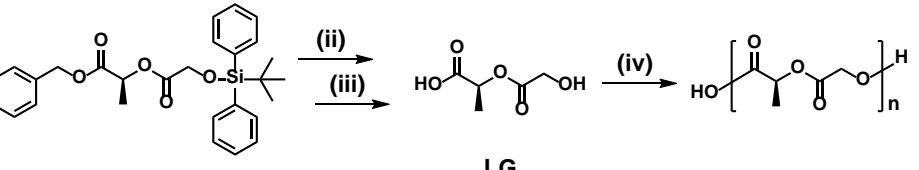

Bn-LG-Si

"segmer"

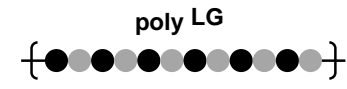

(iii)

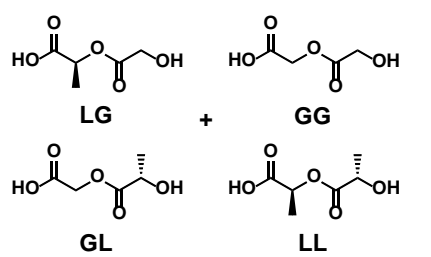

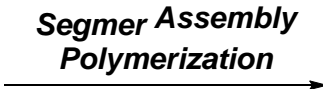
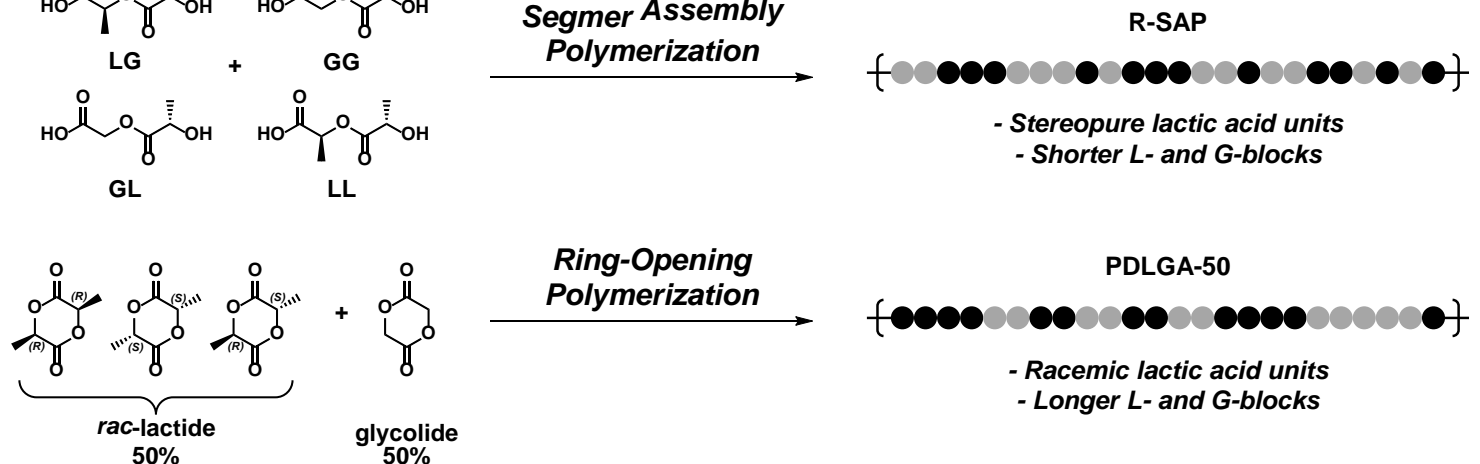

Ring-Opening Polymerization

PDLGA-50

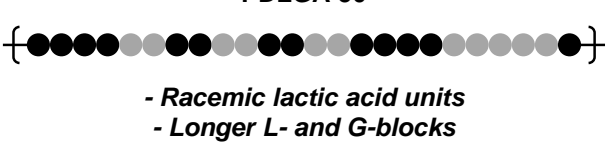

Scheme 1. Synthesis of periodic PLGA copolymers using segmer assembly polymerization (SAP) method along with random analogs synthesized by SAP and ringopening polymerization (ROP).

Table 1.PLGA characterization data

\begin{tabular}{lccccc}
\multicolumn{1}{c}{ Polymer } & $\mathrm{M}_{\mathrm{n}}{ }^{\mathrm{a}}(\mathrm{kDa})$ & $\mathrm{M}_{\mathrm{w}}{ }^{\mathrm{a}}(\mathrm{kDa})$ & $\mathrm{Ð}^{\mathrm{a}}$ & $\mathrm{T}_{\mathrm{g}}\left({ }^{\circ} \mathrm{C}\right)^{\mathrm{b}}$ & ratio L: $^{\mathrm{c}}$ \\
\hline Poly LG & 23.5 & 30.0 & 1.3 & 44 & $50: 50$ \\
Poly L ${ }_{\text {rac }} \mathrm{G}$ & 30.8 & 44.4 & 1.4 & 49 & $50: 50$ \\
Poly GLG & 21.6 & 28.3 & 1.3 & 45 & $34: 66$ \\
Poly GLLG & 18.7 & 25.1 & 1.3 & 45 & $50: 50$ \\
R-SAP & 24.1 & 33.3 & 1.4 & 46 & $44: 56$ \\
PDLGA-50 & 30.7 & 38.4 & 1.3 & 48 & $51: 49$ \\
PLLGA-50 & 23.9 & 38.8 & 1.6 & 47 & $54: 46$ \\
PDLGA-65 & 28.3 & 39.2 & 1.4 & 47 & $65: 35$
\end{tabular}

${ }^{a}$ Determined by size exclusion chromatography in THF relative to polystyrene standards. ${ }^{\mathrm{b}}$ Obtained in the second heating cycle at $10{ }^{\circ} \mathrm{C} / \mathrm{min}$. ${ }^{\mathrm{c}}$ Results based on ${ }^{1} \mathrm{HNMR}$ spectroscopy and presented as the ratio of the lactic (L) and glycolic(G) units. 


\subsection{Characterization}

The copolymers were characterized by ${ }^{1} \mathrm{H}$ NMR, ${ }^{13} \mathrm{C}$ NMR,and MALDI-ToF mass spectrometry(Figures 1, 2, S1-2). As we have previously described in detail the assignment of ${ }^{1} \mathrm{H}$ and ${ }^{13} \mathrm{C}$ NMR spectra of these polymers[26], we will comment on only few key features. Most importantly, we note that these sequenced copolymer exhibit unusually sharp and well-resolved ${ }^{1} \mathrm{H}$ NMRpeaks, even for high molecular weight samples. These copolymers have strong sequence-based conformational preferences which result in unique and assignable resonances for each variant. The diastereotopic glycolyl protons are particularly useful in the ${ }^{1} \mathrm{H}$ NMR spectrum. The combination of clear sequence based differences in chemical shift, along with high resolution means sequence mistakes are readily identifiable and quantifiable.

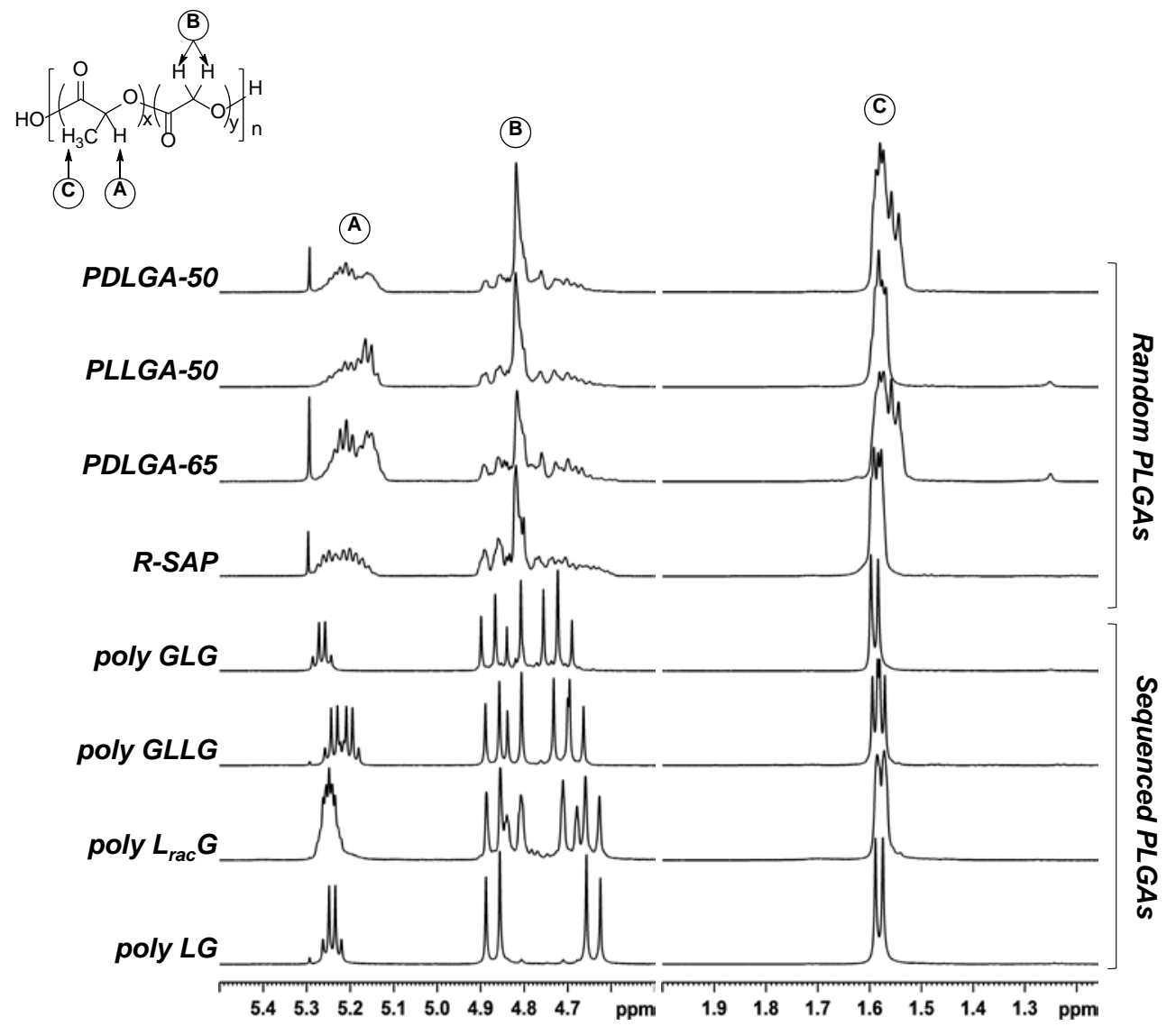

Figure 1. ${ }^{1} \mathrm{H}$ NMR (500 MHz) spectra of sequenced and random PLGAs ( $\delta 5.5-$ 4.5 and $2.0-1.2 \mathrm{ppm}$ ). Labels corresponding to the locations of methine (A), methylene (B), and methyl (C) proton chemical shifts for poly(lactic-co-glycolic acid) are included for reference. 


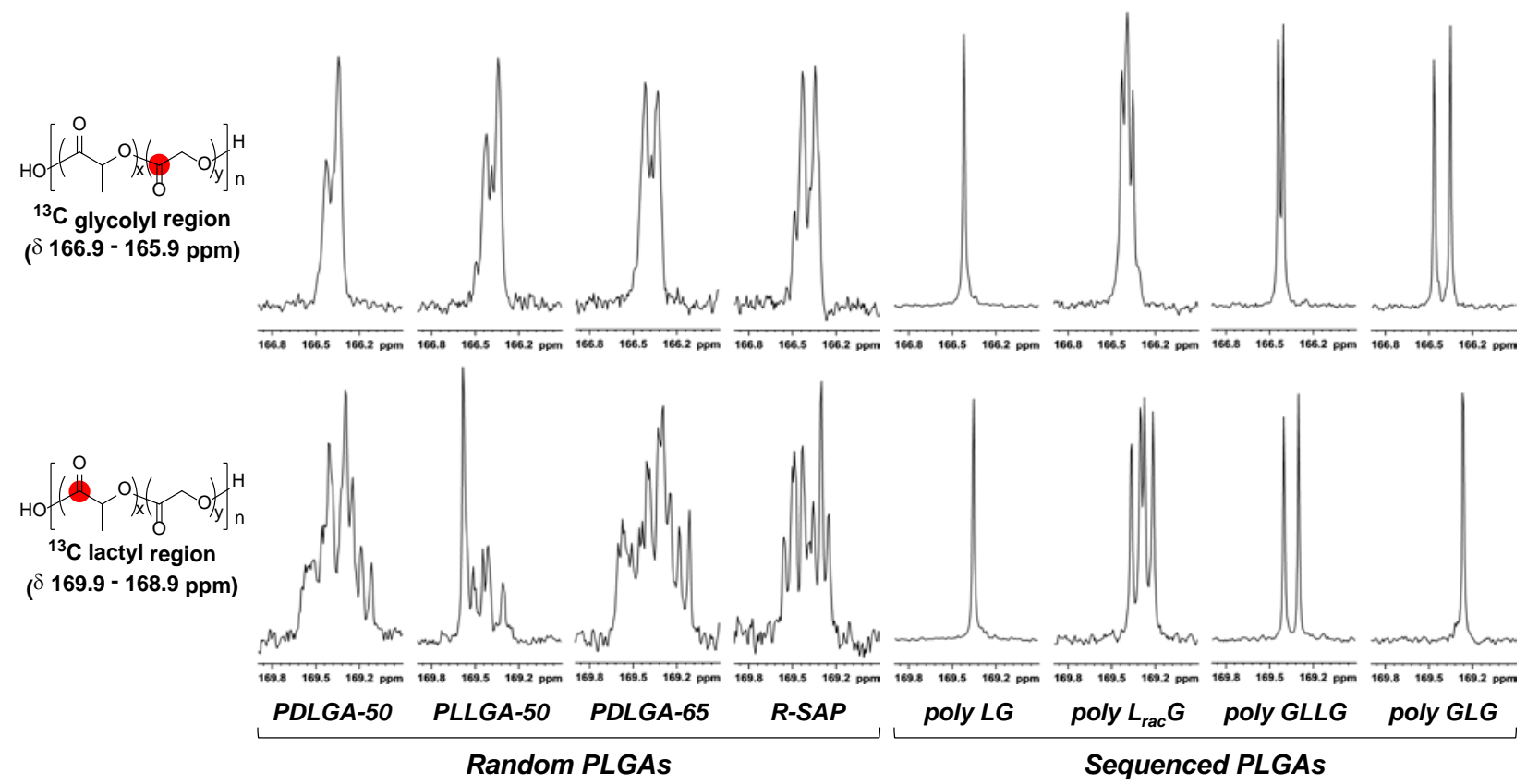

Figure 2. ${ }^{13} \mathrm{C}$ NMR (500 MHz) spectra of glycolyl (top) and lactyl (bottom) carbonyl regions of sequenced and random PLGAs.

MALDI-ToF mass spectra (Figure S1) of these copolymers also confirms the periodic structure of these copolymers as the chain lengths observed are all multiples of the target sequence. With the exception ofPoly GLG the sequenced copolymers, while not perfect, exhibit very small amounts of error and epimerization. The Poly GLGsample, as can be seen in both the ${ }^{1} \mathrm{H}$ and MALDI-ToF spectra does contain a slightly higher rate of sequence errors, primarily consisting of the loss/addition of G or LG units. The rate is fairly low, with no more than one error per chain seen for chains in (GLG) 10 region of the MALDI-ToF spectrum.We believe the error arises, in this case, from the relatively low stability of the GLG segmer from which it is prepared. Again, it should be noted that the assignment of these NMR spectra has been extensively investigated and is described in detail elsewhere[26]. The ${ }^{1} \mathrm{H}$ NMR spectra of the random copolymers, PDLGA-50, PLLGA-50and R-SAP were also acquired and the actual L:G unit ratio (Table 1) was calculated by integration of the glycolic methylene and lactic methine resonances.

The ${ }^{13} \mathrm{C}$ NMR spectra ofthe carbonyl region of the sequenced and random copolymers are particularly interesting to the current discussion as they highlight the dramatic difference in numbers and types of sequence environments (Figure 2). The random copolymers show a large variety of carbonyl environments, while the sequenced copolymers exhibit only a few. The introduction of stereosequences also increases the complexity of this region, even for the periodic copolymerpoly $\mathbf{L}_{r a c} \mathbf{G}$.

As R-SAP had been prepared from the dimers LL, GG, LG, and GL, we expect that the average $\mathrm{L}$ and $\mathrm{G}$ block lengths are most probably shorter than those in commercial PDLGA-50 and PLLGA-50 produced from the ROP of lactide and glycolide. We were not, surprisingly, able to confirm this hypothesis spectroscopically because we found that the ${ }^{13} \mathrm{C}$ NMR resonances 
of the periodic copolymers produced for this study and others overlapped in a way that precludes a simple interpretation of this region for random mixtures (Figure S3 for an example). We note that the prior assignments of resonances that have been proposed based on ROP copolymer syntheses [41-44]cannot be applied in the case of R-SAP because those analyses were based on the predicted absence of resonances for certain sequences that are not forbidden in the R-SAP case. That being said, it is clear from the observed differences in the glycolyl carbonyl region of PLLGA-50 and R-SAP, that there are significant microstructural differences. These differences should, based on our sequence hypothesis, be reflected in the properties.

\subsection{In vitro swelling of sequenced and random PLGAs}

PLGA performance has been examined in a variety of constructs including microparticles $[39,45]$, films [46, 47] and solid matrices in a variety of geometries [33, 48]. For the current studies, which focus on bulk properties rather than drug release, we have chosen to fabricate the polymers into macroscale cylindrical matrices to increase their relevance to larger implantable devices, e.g., screws and plates. To determine dependence of the uptake of water on sequence, swelling studies were performed on cylindrical pellets ( $3 \times 3 \mathrm{~mm})$, two per polymer, prepared using heated compression molding. For random PLGA, these matrices would be expected to degrade by a bulk hydrolysis mechanism since they are thinner in all dimensions than the critical thickness of $7.4 \mathrm{~cm}$ that has been identified as the transition point between bulk and surface erosion for poly( $\alpha$-hydroxy ester)s [33]. Data for cylindrical constructs similar to those used in this study, prepared using random PLGAs, were reported by von Bukersroda etal., in their efforts to develop improved osteosynthetic devices [49-51].

The samples were exposed to buffer at physiological temperature and pHand their hydrated masswas recorded over an eight-week time period. The size and shape of the hydrated pellets at each time point were documented photographically (Figure 3). Theswelling \% of the duplicate samples was calculated according to Eq. 1 where $m_{0}$ is the initial sample mass and $m_{t}$ is the mass

of thehydratedpellet at time $t$ (Figure 4).

$$
\text { swelling } \%=\frac{m_{t}-m_{0}}{m_{0}} \times 100 \%
$$




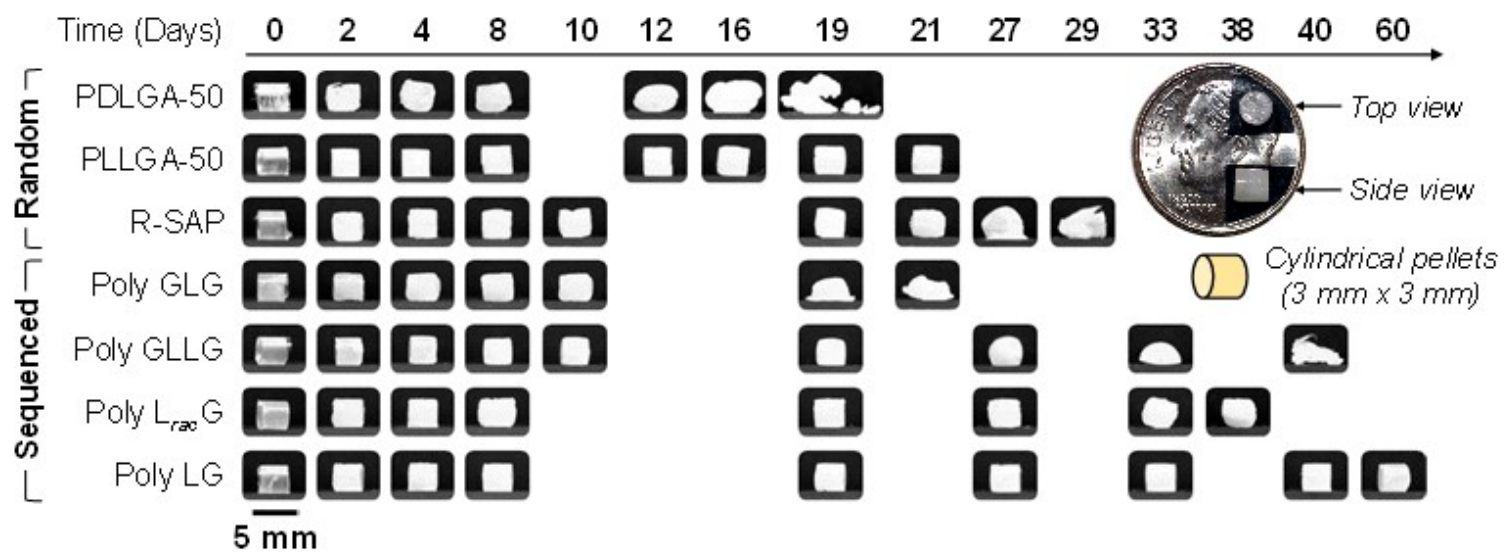

Figure 3.Side-view appearance ofwater-swollen cylindrical pellets as a function of hydrolysis time.
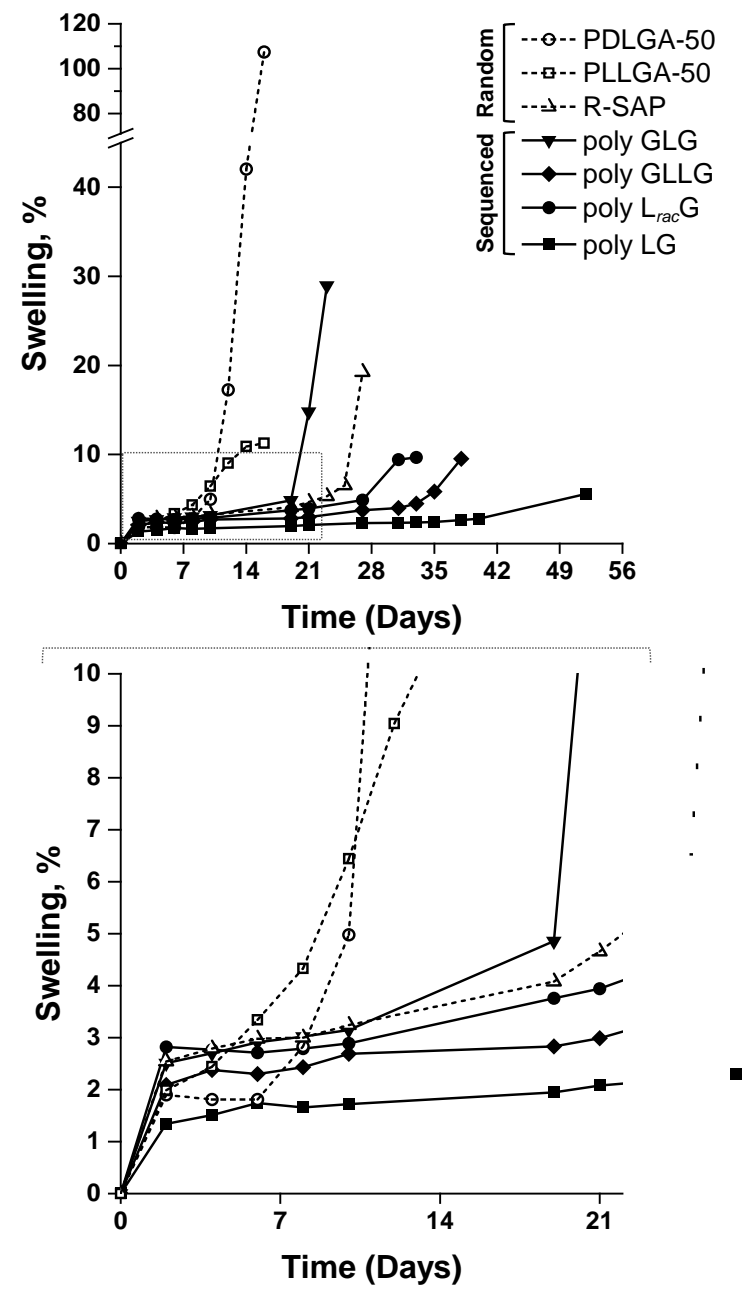

Figure 4.Swelling profiles of sequenced and random PLGAs over 8 weeks(top); enlargementof the first 3-week time period (bottom). Open symbols represent random copolymer controls and closed symbols represent sequenced copolymers. 
In examining the swelling data, it can be seen that upon immersion there was an initial increase in swelling of $\sim 2 \%$ for all polymers during the first two days. The pellet size and morphology remained constant during this time period but gradually changed over the first week depending on the sequence of the PLGA. The onset of swellingfor both random PLGAs, PDLGA-50 and PLLGA-50, occurred during the first week. Interestingly, the morphology of PDLGA-50 changed during this time period and PLLGA-50 remained unchanged.

During the second week, swelling increased from 5\% to 42\% for PDLGA-50; significant swelling and altered shape morphology was also observed. The stereopure random analog PLLGA-50 slowly increased in water content from $6 \%$ to $11 \%$ while retaining its structural integrity. The swelling of sequenced PLGAs remained unchanged during this time period with only R-SAP, the random PLGA analog prepared by SAP, exhibiting slight changes in morphology. After three weeks of immersion, the influence of sequence and stereochemistry was more pronounced. The water content of PDLGA-50 reached its maximum of $107 \%$ and significant sample fracturing was observed. The loss of integrity precluded any further measurements.PLLGA-50 exhibited nominal visual swelling and had a maximum of 11\%prior to sample failure. No significant changes in swelling were observed for poly LG,poly $\mathbf{L}_{\text {rac }} \mathbf{G}$ and poly GLLG, 2\%, 4\% and 3\%, respectively. Noticeable changes in morphology were observed in poly GLG(15\%)and R-SAP(5\%) which were followed by a continual increase in swelling resulting in rupture after 23 days for poly GLG (30\%) and 27 days for R-SAP (20\%). In the time period of week four to five, the morphology of poly GLLG and poly $\mathbf{L}_{\text {rac }}$ Gbegins to change and is accompanied by an increase in swelling, 5-10\% and 4-6\%, respectively, with each sample failing at a maximum swelling of $10 \%$. Throughout this time period, poly LGexhibited minimal changes in swelling and morphology; at 52 days swelling was only $6 \%$.

\subsection{In vitro erosion of sequenced and random PLGAs}

To understand the erosion behavior of sequenced and random PLGAs, mass loss experiments were conducted in parallel to the swelling studies. Cylindrical pellets with a height of $1.5 \mathrm{~mm}$ and width of $3 \mathrm{~mm}$, prepared by heated compression molding were used. These studies were performed under physiological $\mathrm{pH}$ and temperature and mass loss data were obtained on the lyophilized pellets at selected time points over 19 weeks. The size and shape of the lyophilized pellets were recorded photographically and are reported in (Figure 5) and erosion profiles of sequenced and random PLGAs are shown in (Figure 6). The erosion is reported as \% mass loss which was calculated using Eq. 2 where $m_{0}$ is the initial sample mass and $m_{t}$ is the mass of the lyophilized pellet at time $t$.

$$
\% \text { mass loss }=\frac{m_{0}-m_{t}}{m_{0}} \times 100 \%(2)
$$




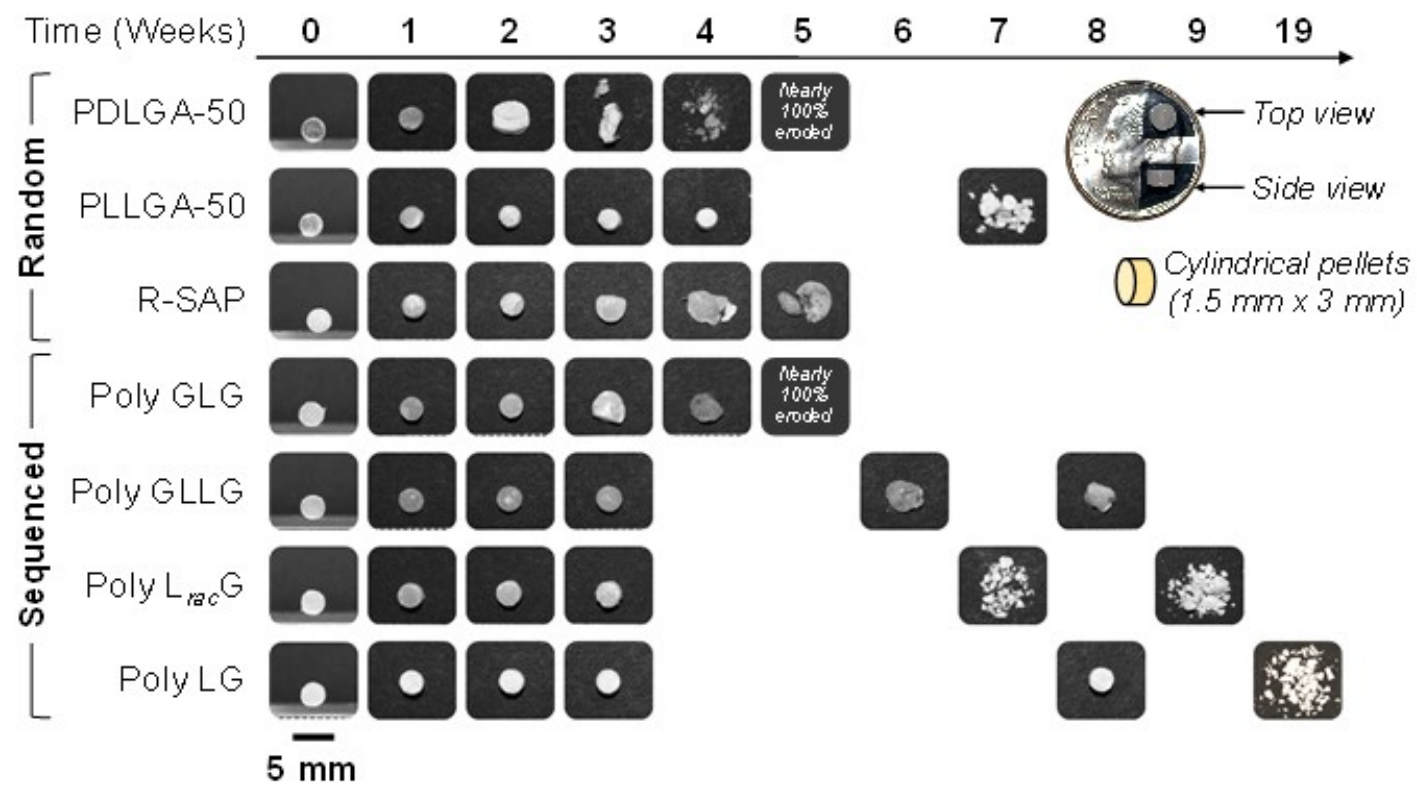

Figure 5. Top-viewappearance of erodedcylindrical pellets, after lyophilization,as a function of hydrolysis time. 

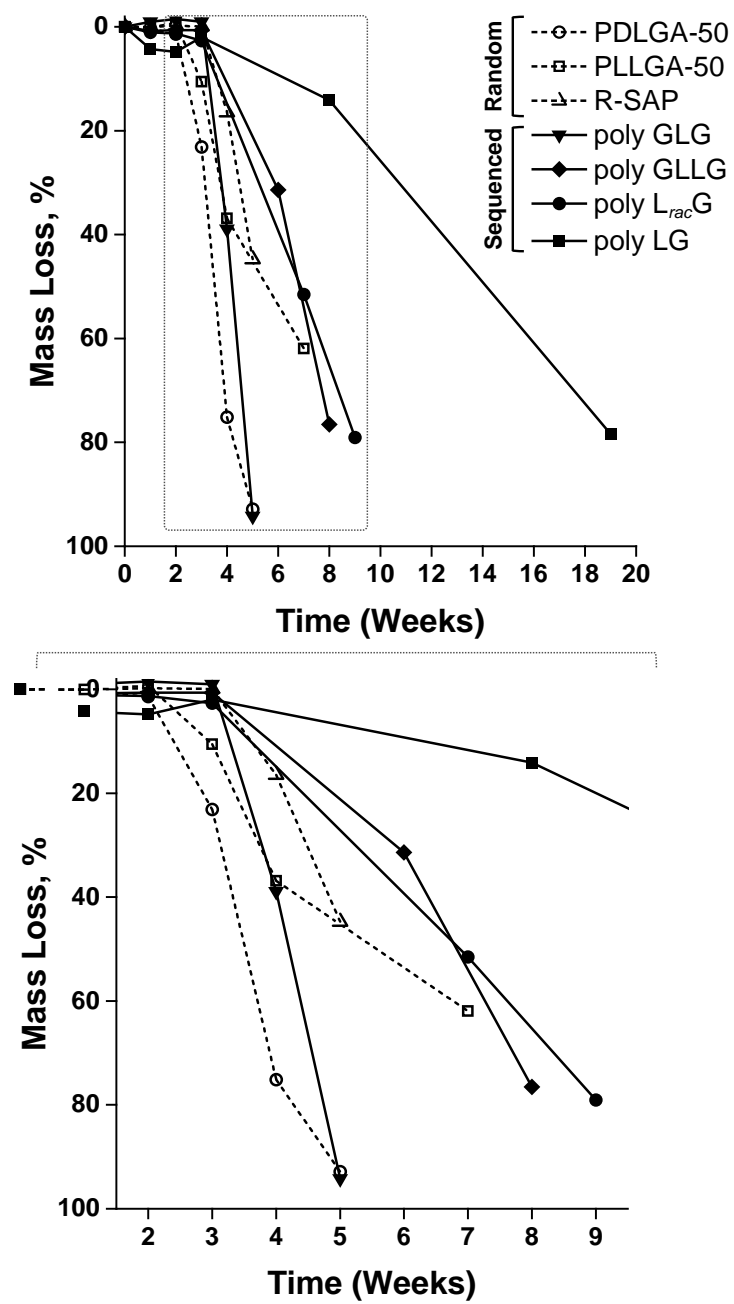

Figure 6. Erosion profiles of sequenced and random PLGAs over 19 weeks (top); enlargement of weeks 2-9 (bottom). Open symbols represent random copolymer controls and closed symbols represent sequenced copolymers.

The mass loss profiles for periodic PLGAs were found to be dramatically different than random PLGAs for a variety of sequences. All samples began with an initial period of stability. After this initial period, the degradation proceeded at a rate that depended on L:G ratio, structural sequence and stereochemistry. For the random PLGAs, PDLGA-50 and PLLGA-50, the onset of erosion occurred at two weeks. The 1:1 LG ratio polymer, PDLGA-50, then lost mass rapidly over the next three weeks before losing structural integrity. The random stereopure analog, PLLGA-50, retained its structural integrity after the onset of erosion for an additional two weeks but lost its structural integrity at week seven, at a62\% mass loss. Interestingly, for PLLGA-50, the erosion rate plateaued between weeks four and seven.

The sequenced PLGAs, with the exception of poly LG,and the random analog prepared by SAPexhibited an onset of erosion during week three. Interestingly, both poly GLG and R-SAP lost their structural integrity at week three and had rapid stages of mass loss throughout weeks four and five. During these time periods, poly GLGlost 39\% of its mass at week four and an 
additional 55\% by week five. R-SAPat the same time points lost $16 \%$ and an additional $28 \%$. Mass loss for poly GLLG and poly $\mathbf{L}_{r a c} \mathbf{G}$ was more gradual. Despite having the same onset of erosion, poly GLLGand poly $\mathbf{L}_{r a c}$ Gretained their structural integrity over weeks four and five but failed at weeks six and seven, respectively. During the time period of weeks three to six for poly GLLG, only $31 \%$ eroded and an additional $45 \%$ eroded after eight weeks. For poly $\mathbf{L}_{\text {rac }} \mathbf{G}$, $49 \%$ of the sample was lostbetween weeks three and seven and an additional $27 \%$ after nine weeks. The erosion profile of poly LG was more linear and structural integrity was maintained over a longer time period; no pronounced onset of erosion wasevident over the time period studied. The structural integrity of poly LGwas retained for eight weeks with only a $14 \%$ mass loss. At the end of the study, poly LG had lost 78\% of the sample's initial mass.

\subsection{Correlated trends in swelling and erosion}
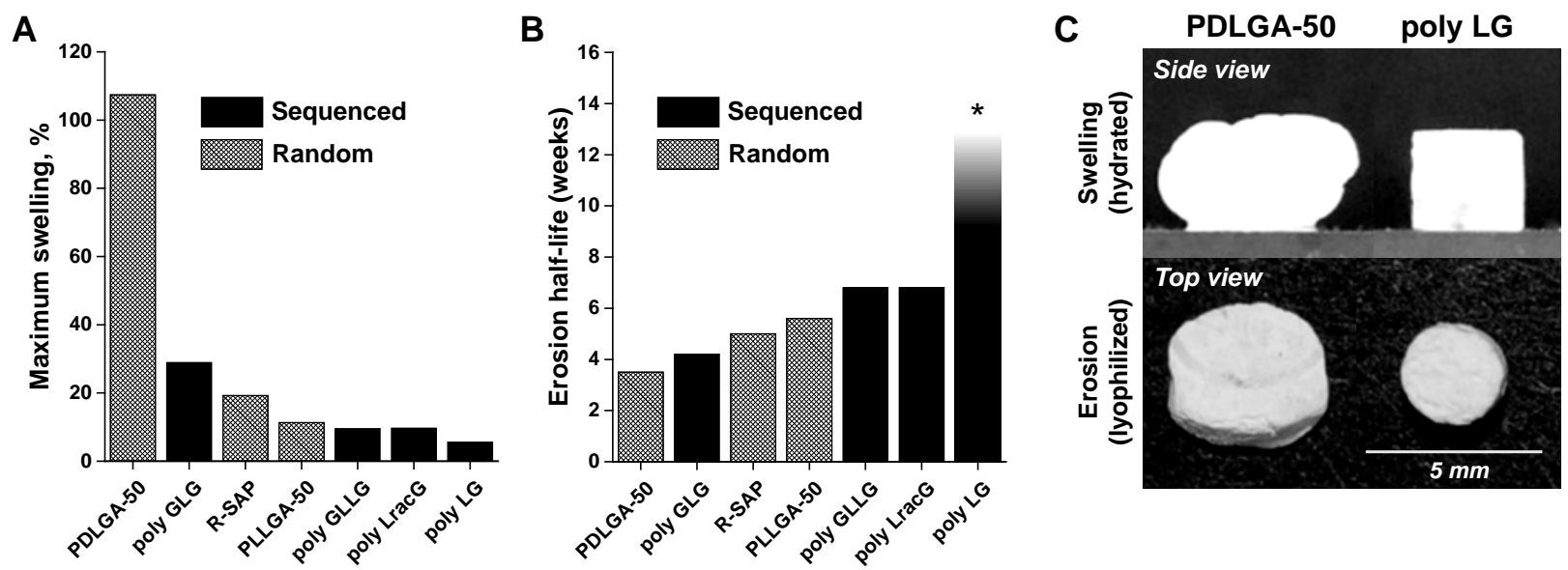

Figure 7.Maximum swelling \% (A) and erosion half-life (B) of sequenced and random PLGAs. *Erosion half-life is approximately 10-14 weeks. Enlarged views (C) ofcylindrical constructs of PDLGA-50 and poly LG in week 2 of degradation highlighting the dramatic sequence-based differences in swelling and erosion behavior.

When the swelling and erosion behaviors of copolymers are compared, there is a clear inverse relationship. Those polymers that exhibited a higher degree of swelling were also observed to erode more quickly (Figure 7). Another important observation that can be made by examining these trends is that both the random PDLGA-50 and poly LG are outliers amongst their analogs. PDLGA-50 swelled by a factor of 3x more than any other sample. On the other end of the spectrum is the alternating copolymer poly LG which erodes 2 x more slowly than any other PLGA examined, including other sequences. The dependence of both processes on stereochemistry can also be seen, with the racemic versions exhibiting more swelling and shorter erosion half-lives than their stereopure analogues.Finally, itis interesting to note that poly GLG which would be expected, based only on the ratio of L:G units, to degrade the quickest, has a half-life that is slightly longer than the random PDLGA-50.

Although the large and repeatable differences in behavior between sequences suggest that the sequence is retained during the degradation process, esters are known to undergo transesterification in the solid state under some conditions. Indeed the synthesis of random 
PLGA has been reported by the thermolysis of PLA with PGA[41]. To probe the possibility of transesterification during hydrolysis in these studies, we collected ${ }^{1} \mathrm{H}$ NMR spectra of the simple alternating stereopure poly LG as a function of degradation (Figure S6). As the NMR spectra for these polymers is exquisitely sensitive to changes in sequence and stereochemistry, any changes would be evident[26].Over 35 days, however, despite evidence of some degradation to form oligomers, there was no observed transesterification nor epimerization of the poly LG structure (FigureS7).

\subsection{Changes in molecular weight and distribution}
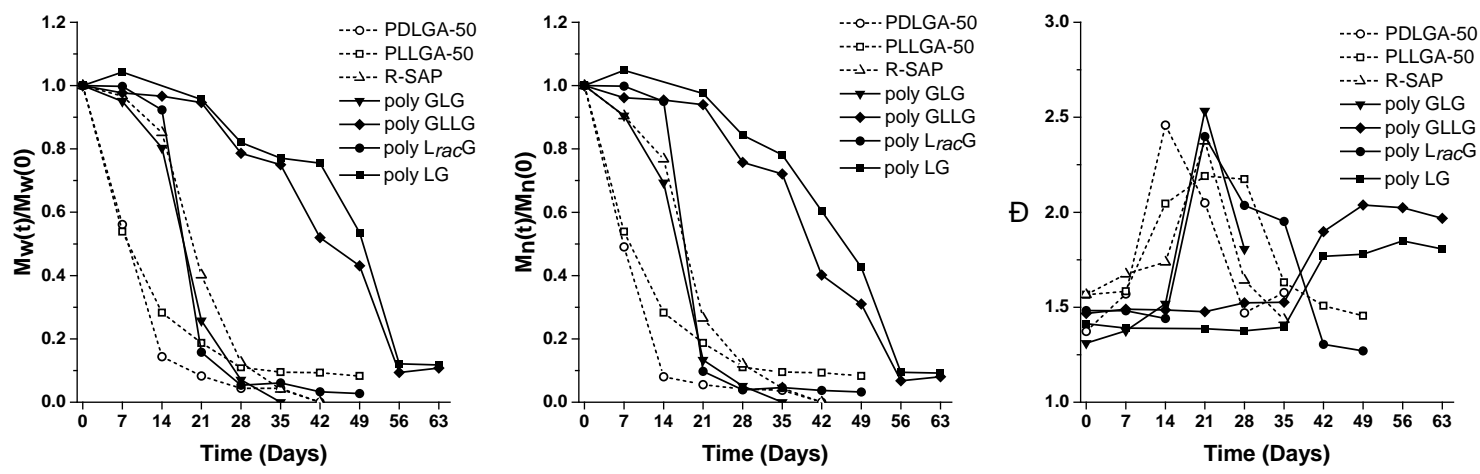

Figure 8. Weight average molecular weight (left); number average molecular weight (center); dispersity (right) ofsequenced and random PLGAs as a function of time. Open symbols represent random copolymer controls and closed symbols represent sequenced copolymers.

The molecular weight profiles for all polymers in this study, normalized relative to the original $\mathrm{M}_{\mathrm{w}}$ and $\mathrm{M}_{\mathrm{n}}$, along with the dispersity data are plotted in Figure 8.The molecular weight loss profiles for random PLGAs, PDLGA-50 and PLLGA-50, decreasedrapidly with timewhich is typical of 50:50 ratio random PLGAs [52]. In contrast, R-SAP, poly GLG and poly $\mathbf{L}_{\text {rac }}$ Gdid not exhibit significant molecular weight loss until after $7 \mathrm{~d}$. Poly LG and poly GLLGretain theirinitial molecular weights for 3 weeks.

Polymer chain dispersity $(\theta)$ was also found to depend on monomer order and stereochemistry.PDLGA-50, R-SAP,poly $\mathbf{L}_{r a c} \mathbf{G}$ and poly GLG exhibit a sharp increase in dispersity by week 3. During this time period PLLGA-50 also increases but at a slower rate. The dispersities of poly LG and poly GLLG remain constant over $35 \mathrm{~d}$, and increase gradually over the following 4 weeks. 


\subsection{Surface water contact angle}

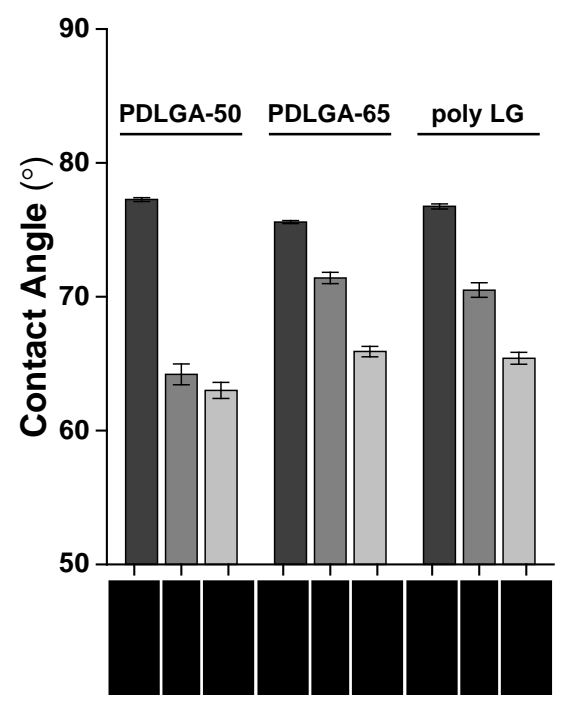

Figure 9.Average water contact angles from unexposed films (initial) and films exposed to physiological conditions over 8 days measured in their hydrated (post-wet) and lyophilized (post-dry) states.Error bars represent the standard error of the mean.

To determine if there were significant differences in the inherent hydrophilicity of the sequenced and random PLGAs, the surface contact angles of selected samples, PDLGA-50, PDLGA-65andpoly LG, were measured after exposure to hydrolyzing conditions. Thick films (20-30 $\mu \mathrm{m})$ were submerged in PBS buffer at $23^{\circ} \mathrm{C}$ and the hydrated contact angle was monitored over an 8 day time period. The samples were subsequently dried by lyophilization and the dry film contact angles were recorded (Figure 9).

The initial water contact angle for all samples was relatively similar, $76.5 \pm 0.5^{\circ}$ and agrees with previously reported measurements[53,54]. There were no significant changes in the hydrated film contact angles over an8 day time period, with the average contact angles for PDLGA-50, PDLGA-65, and poly LG being $63^{\circ}, 66^{\circ}$ and $65^{\circ}$, respectively (Figure S8). The lyophilized film water contact angles, however, do show initial differences after 1 day of exposure to physiological $\mathrm{pH}$ and temperature (Figure S9). The initial contact angle of PDLGA50 decreases from $77^{\circ}$ to $63^{\circ}$, which then remains unchanged at around $62-63^{\circ}$ for the remaining 8 days of exposure. In contrast, the lyophilized contact angles for PDLGA-65 and poly LG do not change significantly after 1 day of exposure. The change in the lyophilized contact angle for PDLGA-50 and poly LG over 8 days was minimal with the overall averages being $72^{\circ}$ and $71^{\circ}$, respectively. The differences observed between sequences in the lyophilized angles appears to correlate with the amount of film degradation — the slower degrading samples maintain their surface hydrophobicity longer. 


\section{Discussion}

The ultimate goal of this work and that of many researchers in bioengineering is to be able to control the degradation of implantable/injectable polymers. While the architecture, i.e., size, shape, etc., of particular matrices contributes significantly to their behavior[55, 56], the chemical composition of the material from which they are made is ultimately responsible for determining the degradation mechanism, profile, release rate of encapsulated drugs, and mechanical properties. The most widely adopted strategy for changing polymer composition, and thereby tuning properties, is to add new or modify the current monomers. While this approach works well for materials that will not be employed in biomedical applications, the use of new monomers in a biomaterial presents significant challenges as all of the degradation products must be non-toxic and clearable.

Our approach to composition control, which avoids the introduction of new chemical entities, is to adopt nature's own solution to this problem: use the same monomers but change the order. Despite the obvious nature of this idea, the synthetic challenges in making sequenced copolymers have long inhibited the exploration of this strategy. There are only isolated, nearly anecdotal, studies relating bulk properties to sequence outside of amino and nucleic acid polymers[11-14]. We have focused our research on PLGA due to the ubiquity of its use and because the poor match of PLGA properties with those required for particular applications has been cited as the justification for the synthesis of a multitude of polymers based on alternate monomers[56-58].It was our hypothesis, one now supported by the results herein, that sequence control may offer an alternate approach to adjusting PLGA properties to those required for particular applications.

Swelling and erosion are both intrinsically involved in degradation[33]. While our data conclusively demonstrate that both behaviors depend on sequence, the reason for the correlationis not immediately apparent. One possible explanation, especially given the dramatic differences in swelling, is that the properties correspond to sequence-based variations in hydrophilicity. Although not a true measure of intrinsic hydrophilicity, the fact that the surface contact angle of PDLGA-50, which necessarily possesses runs of L and G, is nearly the same the simple alternatingpoly LG, suggests that sequence-based differences in the interaction with water are minimal. More convincingly, we find that the water uptake in the first 3 days of the swelling experiment was nearly the same for all samples. These results suggest that the differences seen are nota function of the initial interaction with water but rather come about as a result of chain degradation processes.

The molecular weight loss profile matches the trends that we reported previously [27, 28] and tracks well with the swelling and erosion behaviors. The ROP-random copolymers degrade more quickly than either the R-SAP or sequenced copolymers. The dispersity trends are also interesting as the dispersity increase correlates in time with the onset of significant swelling in all of the polymers.

Stereosequence also plays a key role in degradation. Both stereopure PLLGA-50 and poly LG retain their structural integrity over a slightly longer time period than their racemic analogs, 
PDLGA-50 and poly $\mathbf{L}_{r a c} \mathbf{G}$, respectively. While it is known that crystallinity plays a role in thedifferences between the random copolymers [59, 60], the sequenced copolymers do not appear to crystallize to a significant extent, even during degradation [27]. A fuller understanding of this phenomenon will require further study outside the scope of the current investigation.

Overall, we hypothesize that the differences in hydrolysis profile, both in swelling and erosion, are due primarily to the variation in kinetic rates of cleavage of the L-L, L-G, G-L and G-G linkages (Figure 10). This rate difference has long been established in the random copolymer literature and has been used to explain, for example, the increase in $\mathrm{L}$ to $\mathrm{G}$ ratio during the hydrolysis period[45, 59, 61, 62]. The observed molecular weight behavior is consistent as PDLGA-50, which has the full spectrum of linkages, exhibited a faster drop in molecular weight as a function of hydrolysis time than did the isomeric poly LG, which comprises only L-G and G-L linkages. The dispersity behavior of these copolymers also correlates as rapid cleavage of G-G linkages would be expected to result in earlier increases in

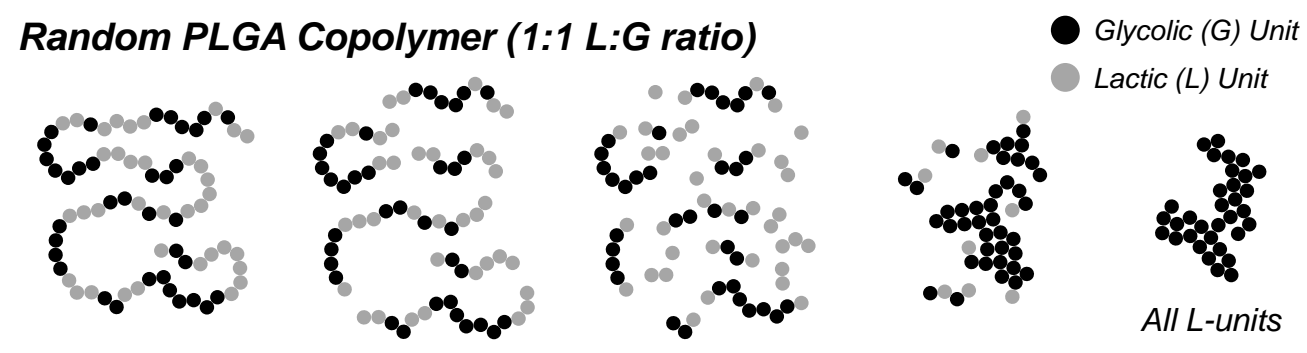

Fast hydrolysis

Slow hydrolysis

Sequenced PLGA Copolymer (1:1 L:G ratio)
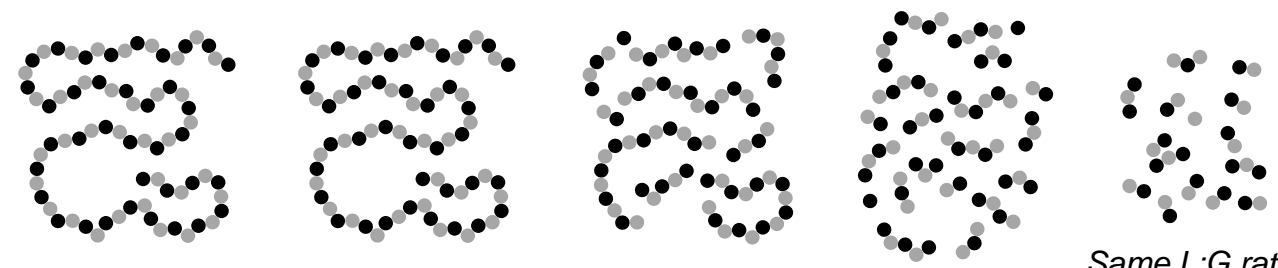

Same $L: G$ ratio

Steady hydrolysis rate

polymer chain dispersity.

Figure 10.Differences in the rates of hydrolysis for random and sequenced PLGA copolymers with the same $\mathrm{L}: \mathrm{G}$ ratio.

In the current study, thevariation in cleavage rates would be expected to produce significant differences in species population within the pellets which we propose leads to the observed differences in degradation profile. The rate of chain cleavage and concomitant generation of acidic oligomers and monomers would be expected to correlate with the frequency of G-G linkages within the chain-a larger percentage of G-G linkages should lead to higher localconcentrations of hydrolytic products within the construct. Although the higher local concentrations of acidic species would be expected to autocatalytically enhance interior 
degradation and polymer erosion[63-65], this effect alone does not explain the swelling behavior. In addition, we propose that osmotic pressure may play an important role in this case because the degradation products are likely produced more rapidly than they are released [66-68]. Under these circumstances a concentration gradient could drive the osmotic uptake of water to give swelling that correlates with the number ofentrapped products[33]. While swelling should eventually facilitate clearance, thereby relieving osmotic pressure, the constructs appear to lose both structural integrity and morphological stability before such an equilibrium is reached. This hypothesis is consistent with the observation that pellets with identical compositions but higher numbers of G-G linkages degrade much faster andexhibit dramatic morphological changes.

\section{Conclusions}

We have demonstrated that swelling and erosion, which are both key properties related to potential applications of PLGAs, depend dramatically on the sequence of the $L$ and $G$ monomers. Specifically, as the number of G-G linkages is decreased, the degree of swelling is diminished and the erosion is slowed. This dependence on sequence allows for the tuning of the hydrolytic profile without additives or other comonomers. Future work will focus on both improving our understanding of the dependence of hydrolysis on sequence and on studies that test the in vivo performance of these materials.

\section{Acknowledgements}

This work was supported by funding from the National Science Foundation [CHE-1410119]. We would like to thank the lab of Dr. Lei Li of the University of Pittsburgh, Department of Chemical \& Petroleum Engineering and the lab of Dr. Kevin Noonan of Carnegie Mellon University, Department of Chemistry for assistance with instrumentation.

\section{References}

[1] Gentile, P, Chiono, V, Carmagnola, I, Hatton, PV, An overview of poly(lactic-co-glycolic) acid (PLGA)-based biomaterials for bone tissue engineering, International Journal of Molecular Sciences, 15 (2014) 3640-3659.

[2] Mu, L, Feng, SS, A novel controlled release formulation for the anticancer drug paclitaxel (Taxol®): PLGA nanoparticles containing Vitamin E TPGS, Journal of Controlled Release, 86 (2003) 33-48.

[3] Fonseca, C, Simões, S, Gaspar, R, Paclitaxel-loaded PLGA nanoparticles: Preparation, physicochemical characterization and in vitro anti-tumoral activity, Journal of Controlled Release, 83 (2002) 273-286.

[4] Derakhshandeh, K, Erfan, M, Dadashzadeh, S, Encapsulation of 9-nitrocamptothecin, a novel anticancer drug, in biodegradable nanoparticles: Factorial design, characterization and release kinetics, European Journal of Pharmaceutics and Biopharmaceutics, 66 (2007) 34-41.

[5] Ulery, BD, Nair, LS, Laurencin, CT, Biomedical applications of biodegradable polymers, Journal of polymer science. Part B, Polymer physics, 49 (2011) 832-864.

[6] Tiainen, J, Soini, Y, Törmälä, P, Waris, T, Ashammakhi, N, Self-reinforced poly(lactide)/poly(glycolide) $80 / 20$ screws take more than $1 \frac{1}{2}$ years to resorb in rabbit cranial bone, Journal of Biomedical Materials Research Part B: Applied Biomaterials, 70B (2004) 4955. 
[7] Pan, Z, Ding, JD, Poly(lactide-co-glycolide) porous scaffolds for tissue engineering and regenerative medicine, Interface Focus, 2 (2012) 366-377.

[8] Wang, Y, Wen, Q, Choi, SH, FDA's regulatory science program for generic PLA/PLGAbased drug products, American Pharmaceutical Review, (2016).

[9] Makadia, HK, Siegel, SJ, Poly(lactic-co-glycolic acid) (PLGA) as biodegradable controlled drug delivery carrier, Polymers, 3 (2011) 1377-1397.

[10] Rothstein, SN, Little, SR, A "tool box" for rational design of degradable controlled release formulations, Journal of Materials Chemistry, 21 (2011) 29-39.

[11] Lutz, J-F, Ouchi, M, Liu, DR, Sawamoto, M, Sequence-controlled polymers, Science, 341 (2013).

[12] Lutz, J-F, Ouchi, M, Sawamoto, M, Meyer, TY, Sequence-controlled polymers: Synthesis, self-assembly, and properties, ACS Symposium Series, American Chemical Society, 2014, pp. 408.

[13] Badi, N, Lutz, J-F, Sequence control in polymer synthesis, Chemical Society Reviews, 38 (2009) 3383-3390.

[14] Badi, N, Chan-Seng, D, Lutz, J-F, Microstructure control: An underestimated parameter in recent polymer design, Macromolecular Chemistry and Physics, 214 (2013) 135-142.

[15] Norris, BN, et al., Sequence matters: Modulating electronic and optical properties of conjugated oligomers via tailored sequence, Macromolecules, 46 (2013) 1384-1392.

[16] Stayshich, RM, Weiss, RM, Li, J, Meyer, TY, Periodic incorporation of pendant hydroxyl groups in repeating sequence PLGA copolymers, Macromolecular Rapid Communications, 32 (2011) 220-225.

[17] Weiss, RM, Jones, EM, Shafer, DE, Stayshich, RM, Meyer, TY, Synthesis of repeating sequence copolymers of lactic, glycolic, and caprolactic acids, Journal of Polymer Science Part A-Polymer Chemistry, 49 (2011) 1847-1855.

[18] Weiss, RM, Short, AL, Meyer, TY, Sequence-controlled copolymers prepared via entropydriven ring-opening metathesis polymerization, ACA Macro Letters, 4 (2015) 1039-1043. [19] Zhang, S, Hutchison, GR, Meyer, TY, Sequence effects in conjugated donor-acceptor trimers and polymers, Macromolecular Rapid Communications, (2016) 882-887.

[20] Fernández, J, Etxeberria, A, Sarasua, J-R, Synthesis, structure and properties of poly(llactide-co-epsilon-caprolactone) statistical copolymers, Journal of the Mechanical Behavior of Biomedical Materials, 9 (2012) 100-112.

[21] Fernández, J, Etxeberria, A, Sarasua, J-R, In vitro degradation studies and mechanical behavior of poly(epsilon-caprolactone-co-delta-valerolactone) and poly(epsilon-caprolactone-coL-lactide) with random and semi-alternating chain microstructures, European Polymer Journal, 71 (2015) 585-595.

[22] Fernández, J, Etxeberria, A, Sarasua, J-R, Effects of repeat unit sequence distribution and residual catalyst on thermal degradation of poly(L-lactide/epsilon-caprolactone) statistical copolymers, Polymer Degradation and Stability, 98 (2013) 1293-1299.

[23] Fernández, J, Etxeberria, A, Ugartemendia, JM, Petisco, S, Sarasua, J-R, Effects of chain microstructures on mechanical behavior and aging of a poly(L-lactide-co-epsilon-caprolactone) biomedical thermoplastic-elastomer, Journal of the Mechanical Behavior of Biomedical Materials, 12 (2012) 29-38.

[24] Fernández, J, Larrañaga, A, Etxeberria, A, Sarasua, J-R, Tensile behavior and dynamic mechanical analysis of novel poly(lactide/delta-valerolactone) statistical copolymers, Journal of the Mechanical Behavior of Biomedical Materials, 35 (2014) 39-50. 
[25] Fernández, J, Larrañaga, A, Etxeberría, A, Sarasua, J-R, Effects of chain microstructures and derived crystallization capability on hydrolytic degradation of poly(L-lactide/epsiloncaprolactone) copolymers, Polymer Degradation and Stability, 98 (2013) 481-489.

[26] Stayshich, RM, Meyer, TY, New insights into poly(lactic-co-glycolic acid) microstructure: Using repeating sequence copolymers to decipher complex NMR and thermal behavior, Journal of the American Chemical Society, 132 (2010) 10920-10934.

[27] Li, J, Rothstein, SN, Little, SR, Edenborn, HM, Meyer, TY, The effect of monomer order on the hydrolysis of biodegradable poly(lactic-co-glycolic acid) repeating sequence copolymers, Journal of the American Chemical Society, 134 (2012) 16352-16359.

[28] Li, J, Stayshich, RM, Meyer, TY, Exploiting sequence to control the hydrolysis behavior of biodegradable PLGA copolymers, Journal of the American Chemical Society, 133 (2011) 69106913.

[29] Sun, S, et al., Episcleral drug film for better-targeted ocular drug delivery and controlled release using multilayered poly- $\varepsilon$-caprolactone (PCL), Acta Biomaterialia, 37 (2016) 143-154. [30] Costa, EF, et al., Effects of light exposure, $\mathrm{pH}$, osmolarity, and solvent on the retinal pigment epithelial toxicity of vital dyes, American Journal of Ophthalmology, 155 (2013) 705712, 712.e701.

[31] Rahman, CV, et al., Controlled release of BMP-2 from a sintered polymer scaffold enhances bone repair in a mouse calvarial defect model, J Tissue Eng Regen Med, 8 (2014) 59-66. [32] Anderson, JM, Shive, MS, Biodegradation and biocompatibility of PLA and PLGA microspheres, Advanced Drug Delivery Reviews, 28 (1997) 5-24.

[33] von Burkersroda, F, Schedl, L, Gopferich, A, Why degradable polymers undergo surface erosion or bulk erosion, Biomaterials, 23 (2002) 4221-4231.

[34] Gopferich, A, Mechanisms of polymer degradation and erosion, Biomaterials, 17 (1996) 103-114.

[35] Gopferich, A, Polymer bulk erosion, Macromolecules, 30 (1997) 2598-2604.

[36] Gopferich, A, Erosion of composite polymer matrices, Biomaterials, 18 (1997) 397-403.

[37] Gopferich, A, Polymer degradation and erosion: Mechanisms and applications, European Journal of Pharmaceutics and Biopharmaceutics, 42 (1996) 1-11.

[38] Gopferich, A, Tessmar, J, Polyanhydride degradation and erosion, Advanced Drug Delivery Reviews, 54 (2002) 911-931.

[39] Gasmi, H, Danede, F, Siepmann, J, Siepmann, F, Does PLGA microparticle swelling control drug release? New insight based on single particle swelling studies, Journal of Controlled Release, 213 (2015) 120-127.

[40] Takizawa, K, Nulwala, H, Hu, J, Yoshinaga, K, Hawker, CJ, Molecularly defined (L)-lactic acid oligomers and polymers: Synthesis and characterization, Journal of Polymer Science Part A: Polymer Chemistry, 46 (2008) 5977-5990.

[41] Gao, Q, Lan, P, Shao, H, Hu, X, Direct synthesis with melt polycondensation and microstructure analysis of poly(L-lactic acid-co-glycolic acid), Polymer Journal, 34 (2002) 786793.

[42] Qian, H, Wohl, AR, Crow, JT, Macosko, CW, Hoye, TR, A strategy for control of "random" copolymerization of lactide and glycolide: Application to synthesis of PEG-b-PLGA block polymers having narrow dispersity, Macromolecules, 44 (2011) 7132-7140.

[43] Kasperczyk, J, Microstructural analysis of poly[(L,L-lactide)-co-(glycolide)] by $1 \mathrm{H}$ and $13 \mathrm{C}$ NMR spectroscopy, Polymer, 37 (1996) 201-203. 
[44] Kreiser-Saunders, I, Kricheldorf, HR, Polylactones, 39. Zn lactate-catalyzed copolymerization of L-lactide with glycolide or $\varepsilon$-caprolactone, Macromolecular Chemistry and Physics, 199 (1998) 1081-1087.

[45] Park, TG, Degradation of poly(lactic-co-glycolic acid) microspheres - Effect of copolymer composition, Biomaterials, 16 (1995) 1123-1130.

[46] Vey, E, et al., Degradation kinetics of poly(lactic-co-glycolic) acid block copolymer cast films in phosphate buffer solution as revealed by infrared and raman spectroscopies, Polymer Degradation and Stability, 96 (2011) 1882-1889.

[47] Vey, E, et al., The impact of chemical composition on the degradation kinetics of poly(lactic-co-glycolic) acid copolymers cast films in phosphate buffer solution, Polymer Degradation and Stability, 97 (2012) 358-365.

[48] Keles, H, Naylor, A, Clegg, F, Sammon, C, Investigation of factors influencing the hydrolytic degradation of single PLGA microparticles, Polymer Degradation and Stability, 119 (2015) 228-241.

[49] Eglin, D, Alini, M, Degradable polymeric materials for osteosynthesis: Tutorial, European Cells \& Materials, 16 (2008) 80-91.

[50] Ciccone II, WJ, Motz, C, Bentley, C, Tasto, JP, Bioabsorbable implants in orthopaedics: New developments and clinical applications, The Journal of the American Academy of Orthopaedic Surgeons, 9 (2001) 280-288.

[51] Athanasiou, KA, Agrawal, CM, Barber, FA, Burkhart, SS, Orthopaedic applications for PLA-PGA biodegradable polymers, Arthroscopy, 14 (1998) 726-737.

[52] Siepmann, J, Elkharraz, K, Siepmann, F, Klose, D, How autocatalysis accelerates drug release from PLGA-based microparticles: A quantitative treatment, Biomacromolecules, 6 (2005) 2312-2319.

[53] Liu, Y, et al., Surface hydrophobicity of microparticles modulates adjuvanticity, Journal of Materials Chemistry B, 1 (2013) 3888-3896.

[54] Mei, FF, et al., Synthesis and characterization of biodegradable poly(lactic-co-glycolic acid), Journal of Macromolecular Science Part B-Physics, 54 (2015) 562-570.

[55] Fredenberg, S, Wahlgren, M, Reslow, M, Axelsson, A, The mechanisms of drug release in poly(lactic-co-glycolic acid)-based drug delivery systems- a review, International Journal of Pharmaceutics, 415 (2011) 34-52.

[56] Kamaly, N, Yameen, B, Wu, J, Farokhzad, OC, Degradable controlled-release polymers and polymeric nanoparticles: Mechanisms of controlling drug release, Chemical Reviews, 116 (2016) 2602-2663.

[57] Seyednejad, H, Ghassemi, AH, van Nostrum, CF, Vermonden, T, Hennink, WE, Functional aliphatic polyesters for biomedical and pharmaceutical applications, Journal of Controlled Release, 152 (2011) 168-176.

[58] Xu, XJ, Sy, JC, Shastri, VP, Towards developing surface eroding poly(alpha-hydroxy acids), Biomaterials, 27 (2006) 3021-3030.

[59] Li, SM, Garreau, H, Vert, M, Structure-property relationships in the case of the degradation of massive poly( $\alpha$-hydroxy acids) in aqueous media, Journal of Materials Science: Materials in Medicine, 1 (1990) 131-139.

[60] Saha, SK, Tsuji, H, Hydrolytic degradation of amorphous films of L-lactide copolymers with glycolide and d-lactide, Macromolecular Materials and Engineering, 291 (2006) 357-368. 
[61] López-Santos, C, Terriza, A, Portolés, J, Yubero, F, González-Elipe, AR, Physiological degradation mechanisms of PLGA membrane films under oxygen plasma treatment, The Journal of Physical Chemistry C, 119 (2015) 20446-20452.

[62] Miller, RA, Brady, JM, Cutright, DE, Degradation rates of oral resorbable implants (polylactates and polyglycolates): Rate modification with changes in PLA/PGA copolymer ratios, Journal of Biomedical Materials Research, 11 (1977) 711-719.

[63] Schadlich, A, Kempe, S, Mader, K, Non-invasive in vivo characterization of microclimate $\mathrm{pH}$ inside in situ forming PLGA implants using multispectral fluorescence imaging, Journal of Controlled Release, 179 (2014) 52-62.

[64] Zolnik, BS, Burgess, DJ, Effect of acidic pH on PLGA microsphere degradation and release, Journal of Controlled Release, 122 (2007) 338-344.

[65] Fu, K, Pack, DW, Klibanov, AM, Langer, R, Visual evidence of acidic environment within degrading poly(lactic-co-glycolic acid) (PLGA) microspheres, Pharmaceutical Research, 17 (2000) 100-106.

[66] Rothstein, SN, Federspiel, WJ, Little, SR, A simple model framework for the prediction of controlled release from bulk eroding polymer matrices, Journal of Materials Chemistry, 18 (2008) 1873-1880.

[67] Rothstein, SN, Federspiel, WJ, Little, SR, A unified mathematical model for the prediction of controlled release from surface and bulk eroding polymer matrices, Biomaterials, 30 (2009) 1657-1664.

[68] Brunner, A, Mader, K, Gopferich, A, pH and osmotic pressure inside biodegradable microspheres during erosion, Pharmaceutical Research, 16 (1999) 847-853. 